\title{
REVIEW
}

\section{Advance in Functional Restoration of Injured Nerve with Low Level Laser and its Utilization in the Dental and Maxillofacial Region}

\author{
Pugen An, Jizhi Zhao* \\ Department of Stomatology, Peking Union Medical College Hospital, \\ Chinese Academy of Medical Sciences \& Peking Union \\ Medical College, Beijing 100730, China
}

Key words: low level laser; nerve injury; functional restoration; dental and maxillofacial surgery

\begin{abstract}
The inferior alveolar nerve and facial nerve are the two most important nerves in the dental and maxillofacial region. The injury to them is one of the major postoperative complications after alveolar surgery and orthognathic surgery. However, recovering the nerve function after injury takes a long time and the recovery effect tends to be unsatisfactory. In recent years, an intensively investigated technique, low level laser which has been applying in assisting the recovery of nerve function, has been gradually proved to be effective in clinically treating postoperative nerve injury. In this article we review in terms of the mechanisms involved in low level laser-assisted functional restoration of nerve injury and its clinical application in the recovery of nerve function in the dental and maxillofacial area as well.
\end{abstract}

$\mathrm{T}$

HE inferior alveolar nerve and facial nerve are the most important nerves in the maxillofacial region. Because of their superficial anatomic location, structure and distribution, they are the most vulnerable to injury in maxillofacial trauma and maxillofacial surgery. Moreover, usually it is difficult and takes a long time for the recovery of nerve function after injury, which brings great discomfort and pain to patients.

In recent years, with the rapid development of stomatology laser medicine, low level laser (LLL) applied in the restoration of nerve function has become a hot topic. There is no clear definition of LLL, but LLL

Received March 15, 2020.

*Corresponding author Tel: 86-10-69156332, E-mail: zhaojizhi@ 126.com is generally defined by most scholars as a particular category of laser light having a wavelength between 600-1100 nm, an output power less than $500 \mathrm{~mW}$, and an energy density less than $50 \mathrm{~J} / \mathrm{cm}^{2}$. Many kinds of LLLs, such as gallium arsenide laser with wavelength of $904 \mathrm{~nm}$, output power of $70 \mathrm{Wpk}$, energy density of $10 \mathrm{~J} / \mathrm{cm}^{2}$, gallium aluminum arsenide carbon dioxide laser with wavelength of $830 \mathrm{~nm}$, output power of $100 \mathrm{~mW}$, energy density of $4 \mathrm{~J} / \mathrm{cm}^{2}$, and carbon dioxide laser with wavelength of $650 \mathrm{~nm}$, output power of $25 \mathrm{~mW}$, energy density of $25 \mathrm{~J} / \mathrm{cm}^{2}$, etc., have been applied to treatment of the dental and maxillofacial diseases such as root canal cleaning and sterilization, therapying peri-implantitis and temporomandibular joint disease, alleviating deep periodontal pocket, and curing oral aphtha ulcer, especially promoting the recovery of nerve function. In this paper, we summarize 
the underlying molecular and histological mechanisms of LLL participating in functional restoration of injured nerve and its clinical application in the recovery of nerve function of the maxillofacial area, aiming to provide insight into this technique.

\section{Mechanism by which LLL promotes the recovery of nerve function}

\section{Molecular biology}

Neurotrophins are a class of secreted proteins produced by nerve-innervated tissues and astrocytes and essential for the growth and survival of neurons. Nerve growth factor (NGF) and brain-derived neurotrophic factor (BDNF), as members of the neurotrophin family, are main regulators of neuronal survival, growth, differentiation, ${ }^{[1]}$ and regeneration. ${ }^{[2,3]}$ Whenever nerves are damaged, they can prevent neurons from dying, improve pathological state of neurons, and accelerate nerve repair. ${ }^{[2,3]}$

The accumulated evidence has proved that the vital role of NGF in promoting the development, maintenance and regeneration of sensory and sympathetic nerves. ${ }^{[4]}$ Gravvanis et al. ${ }^{[5]}$ have confirmed that NGF, as an independent factor, can accelerate hyperplasia of peripheral nerve, and promote repair of injured nerve and recovery of muscle motor potential after nerve injury. Interestingly, in an in vitro study with Schwann cells irradiated by a LLL (gallium-aluminum-arsenide, GaAlAs, wavelength $810 \mathrm{~nm}$, output power $50 \mathrm{~mW}$, energy density $1 \mathrm{~J} / \mathrm{cm}^{2}$ and $4 \mathrm{~J} / \mathrm{cm}^{2}$ ), real time PCR showed NGF expression was significantly increased on day 20 after irradiation. ${ }^{[6]}$ Schwann cells are the glial cells that surround the axon of neurons in the peripheral nervous system, and function in expression and secretion of various neurotrophic factors, ${ }^{[7,8]}$ which suggest LLL may improve and restore function of injured nerve through inducing NGF expression in Schwann cells. ${ }^{[9,10]}$

BDNF as a modulatory factor of pain neurons, which is involved in pain generating, is released by nociceptors. The released BDNF together with glutamate and neuropeptide substance $P$ is fast anterogradely transported to presynaptic terminals to activate spinal dorsal horn secondary neurons to feel pain at last. ${ }^{[11]}$ The increasing levels of BDNF in dorsal root ganglion neurons and spinal dorsal horn, which serves as a modulator at the first synapse of the pain transmission pathway in the spinal dorsal horn, can promote neuropathic pain to happen through BDNF/TrkB signal pathway. ${ }^{[12-14]}$ Oliveira Martins et al. applied LLL therapy (gallium-arsenide, GaAs, wavelength $904 \mathrm{~nm}$, output power $70 \mathrm{Wpk}$, a spot area of $0.1 \mathrm{~cm}^{2}$, frequency $9500 \mathrm{~Hz}$, energy density $6 \mathrm{~J} / \mathrm{cm}^{2}$ ) to treating inferior alveolar nerve injury of experimental rats, and the result showed the LLL could increase pain threshold of the rats and improve nociceptive behavior. Meanwhile, western blot demonstrated that laser irradiation could increase NGF level in the injured inferior alveolar nerve by $53 \%$, and could decrease BDNF level induced by nerve injury by $40 \%$ and lower total pain threshold as well. ${ }^{[15]}$

Those results indicated that LLL may be a useful modality in controlling and reversing pain sensitivity and accelerating neural tissue regeneration by influencing NGF and BDNF expression.

\section{Histocytology}

Neurological damage, as it is known, may contribute to varying degrees of histocytological and morphological changes of neurons, ultimately resulting in neurological dysfunction. Similarly, the functional recovery of neurons after injury is associated with a histocytological and morphological return of injured tissues to normal. Several studies have provided evidence of morphological basis for functional recovery of injured peripheral nerve after application of LLL. They found that LLL ultimately make the functional recovery of peripheral nerve come true specifically through increasing axon number and density, augmenting sheath thickness and nerve fiber diameter of the broken end of injured nerve. ${ }^{[16,17]}$ Buchaim et al. ${ }^{[18]}$ employed LLL (wavelength $830 \mathrm{~nm}$, pulsed, optical power $30 \mathrm{~mW}$, $0.2586 \mathrm{~W} / \mathrm{cm}^{2}, 6.2 \mathrm{~J} / \mathrm{cm}^{2}$, beam area of $0.116 \mathrm{~cm}^{2}$ ) to irradiate the head, middle and tail of the seam respectively of a rat model undergoing buccal branch anastomosis of the facial nerve, three times a week over 5 weeks, morphological analysis performed 35 and 120 days after operation with optical and electron microscope showed the axon number and density of the broken end of injured nerve was greater versus the simple suture group, and the diameter of nerve fibers and the thickness of nerve sheath of the laser group were augmented as well. Meanwhile, the injured facial nerve irradiated by LLL has a higher frequency of whisker movement, which indicated that LLL could improve recovery of motor function of facial nerve. Moreover, LLL excels at accelerating revascularization of dam- 
aged nerve. GaAlAs+GaAlInP diode laser (780 nm, 2 J, $100 \mathrm{~mW}, 40$ seconds used on the day of surgery and $4 \mathrm{~J}, 200 \mathrm{~mW}, 40$ seconds on the subsequent days) was used to irradiate the skin area of injured nerve of sciatic nerve anastomosis rat models, histological examination showed the number of new vessels of the LLL treated region were more compared to the non-laser rats on the third day after surgery, and the tendency became more and more distinct with the time gone by. ${ }^{[19]}$

Besides promoting the healing of injured nerves, LLL irradiation can be directly applied to welding nerves, in which it shows advantages of time-saving, being easy to learn, and more quick restoration of postoperative neurological function in comparison with conventionally used method. For example, in a rabbit model, a diode laser (wavelength $810 \pm 1 \mathrm{~nm}$, pulsed, optical power $0.5 \mathrm{~W}, 15.9 \mathrm{~W} / \mathrm{cm}^{2}, 8.0 \mathrm{~J} / \mathrm{cm}^{2}$ ) used to weld the buccal branch of the facial nerve which was sharply transected, compared with the simple suture group, histopathological analysis of the laser-welding area, showed LLL has an obvious advantage in increasing axon number and density and augmenting sheath thickness and nerve fiber diameter. ${ }^{[16,20-23]}$ Although at 4 weeks the laser-welding area presented a robust inflammatory reaction, at 16 weeks it disappeared completely, which demonstrated laser welding with a biological solder had no a deleterious effect on the nerve axons or fascicles. ${ }^{[20]}$

\section{Clinical application of LLL}

\section{LLL used to improve recovery of inferior alveolar nerve}

The inferior alveolar nerve perforates the lower margin of lateral pterygoid muscle, passes through the mandibular nerve groove accompanied by the inferior alveolar artery and vein, then enters the mandibular canal from the mandibular foramen, which finally manages the bilateral mandibular teeth, periodontal membrane and alveolar bone. The inferior alveolar nerve injury is one of the most common postoperative complications for the removal of impacted mandibular wisdom teeth and sagittal split ramus osteotomy on account of its anatomic location and structure. ${ }^{[24]}$ It is main clinical manifestations are sensory abnormalities and obtuseness mainly involving the lip, gingiva and chin. ${ }^{[25]}$ LLL has been proved to be effective in recovering function of inferior alveolar nerve after injured. For example, in a study of 20 patients receiving mandibular sagittal split osteotomy who were randomly divided into two groups, neurosensory recovery of those who underwent LLL and light-emitted diode radiation $(810 \mathrm{~nm}$, $5 \mathrm{~J} / \mathrm{cm}^{2}$ and $632 \mathrm{~nm}, 2 \mathrm{~J} / \mathrm{cm}^{2}$ ) on 1 day and 2, 3, 7, 14 and 28 days after surgery, in the following four regions: the entrance of the mandibular foramen, along the osteotomy line, the lips, and chin region, was compared with the non-laser group. The results showed that visual analog scale score increased by $25 \%$ at 2 weeks, $21 \%$ at 2 months, and $24 \%$ at 6 months after surgery, brush stroke value was raised by $21.5 \%$ at 2 months and $15.1 \%$ at 6 months, and 2-point discrimination significantly decreased by $15.3 \%$ at 2 weeks and $16.2 \%$ at 2 months. Contact detection was greater after 6 months than the non-laser group although there was no significant intergroup difference, and pinprick test value returned to normal at 45 days, which was faster than the control group. The above results indicated that LLL may facilitate restore function of inferior alveolar nerve. ${ }^{[26]}$ Similarly, in another two randomized clinical trial studies, LLL can effectively improve neurosensory disorders of mandibular nerve damaged during sagittal split osteotomy. ${ }^{[27,28]}$

What is more, LLL could achieve a better healing of affected nerve. Guarini et al. ${ }^{[29]}$ followed up 41 patients of inferior alveolar nerve injury after sagittal split ramus osteotomy treated by LLL, and found $85 \%$ patients of the laser group obtained clinical improvement at 2 years postsurgery, which was greater than that of the placebo group (70\% to $75 \%)$. It is generally recognized that most of patients achieve full functional recovery within six months after nerve impairment. If the impairment continues for more than one year, patients will remain with a permanent impairment of nerve function. ${ }^{[30]}$ Therefore, we advise that to treat the neurosensory impairment of the inferior alveolar nerve due to surgical damage, LLL should start as soon as possible, and continue for as long as completely healed. ${ }^{[31]}$ The earlier LLL starts, the better the results are.

\section{LLL used to treat peripheral facial palsy}

Bell's palsy as a type of peripheral facial palsy, manifested as partial or complete facial palsy, mostly can be attributed to virus infection induced facial nerve edema or inflammation. ${ }^{[32]}$ No definite treatment guideline has been recommended. Corticosteroids, antiviral drugs, facial muscle exercises as well as electri- 
cal stimulation are the most frequently supplied medicines for Bell's palsy. ${ }^{[33-35]}$ Over the years LLL has been applied clinically to treat Bell's palsy and appears to have good results. For example, a three-year-old boy with a sudden onset of facial asymmetry with unknown cause received a gallium aluminum arsenide semiconductor diode laser treatment $\left(70 \mathrm{~mW} ; 17.5 \mathrm{~J} / \mathrm{cm}^{2}\right.$, $780 \mathrm{~nm}$ in the initial 4 sessions and $10 \mathrm{~J} / \mathrm{cm}^{2}, 660 \mathrm{~nm}$ for other 7 sessions) which was applied by direct contact with the facial area in a continuous emission mode for totally 11 sessions, without oral medication or other methods. After 3 weeks of treatment, facial nerve function of the boy was completely recovered. ${ }^{[36]}$ In another clinical study including 46 patients with Bell's palsy administrated with LLLT (GaAIAs infrared laser, $830 \mathrm{~nm}, 100 \mathrm{~mW}, 10 \mathrm{~J} / \mathrm{cm}^{2}$ ) over a period of 6 weeks, facial disability index score which is the most frequently used index for assessment of severity and progression of Bell's palsy, was significantly better than that of the control group. ${ }^{[37]}$ A randomized double blind placebo-controlled trial involving 48 Bell's palsy patients also indicated that LLL treatment versus facial massage and exercises alone can improve both facial disability index and House-Brackmann scale score. ${ }^{[38]}$

In conclusion, LLL could promote the regeneration and recovery of injured nerve and improve the symptoms of patients after nerve injury, and maybe LLL could reliably and effectively serve as an auxiliary tool to promote the functional recovery of injured nerve in orthodontics and alveolar operations. However, the underlying mechanisms by which LLL promotes nerve repair are needed to be clarified.

\section{Conflict of Interest Statement}

The authors declare no conflict of interests.

\section{REFERENCES}

1. Keefe KM, Sheikh IS, Smith GM. Targeting neurotrophins to specific populations of neurons: NGF, BDNF, and NT-3 and their relevance for treatment of spinal cord injury. Int J Mol Sci 2017; 18(3):548. doi: 10.3390/ijms18030548.

2. Patterson SL, Abel T, Deuel TA, et al. Recombinant BDNF rescues deficits in basal synaptic transmission and hippocampal LTP in BDNF knockout mice. Neuron 1996; 16(6):1137-45.

3. Li R, Wu J, Lin Z, et al. Single injection of a novel nerve growth factor coacervate improves structural and functional regeneration after sciatic nerve injury in adult rats. Exp Neurol 2017; 288:1-10. doi: 10.1016/j.expneurol.2016.10.015.

4. Levi-Montalcini R. Growth control of nerve cells by a perotein factor and its antiserum: discovery of this factor may provide new leads to understanding of some neurogenetic processes. Science 1964; 143(3602): 105-10. doi: 10.1126/science.143.3602. 105.

5. Gravvanis AI, Tsoutsos DA, Tagaris GA, et al. Beneficial effect of nerve growth factor-7S on peripheral nerve regeneration through inside-out vein grafts: an experimental study. Microsurgery 2004; 24(5):40815. doi: 10.1002/micr.20055.

6. Yazdani SO, Golestaneh AF, Shafiee A, et al. Effects of low level laser therapy on proliferation and neurotrophic factor gene expression of human schwann cells in vitro. J Photochem Photobiol B 2012; 107:913. doi: 10.1016/j.jphotobiol.2011.11.001.

7. Kim HS, Lee J, Lee DY, et al. Schwann cell precursors from human pluripotent stem cells as a potential therapeutic target for myelin repair. Stem Cell Reports 2017; 8(6):1714-26. doi: 10.1016/j.stemcr. 2017.04.011.

8. Day AGE, Bhangra KS, Murray-Dunning $C$, et al. The effect of hypothermic and cryogenic preservation on engineered neural tissue. Tissue Eng Part C Methods 2017; 23(10):575-82. doi: 10.1089/ten.tec.2017. 0244.

9. Shadiack AM, Sun Y, Zigmond RE. Nerve growth factor antiserum induces axotomy-like changes in neuropeptide expression in intact sympathetic and sensory neurons. J Neurosci 2001; 21(2):363-71. doi: 10.1523/JNEUROSCI.21-02-00363.2001.

10. Chen ZW, Wang MS. Effects of nerve growth factor on crushed sciatic nerve regeneration in rats. Microsurgery 1995; 16(8):547-51. doi: 10.1002/ micr.1920160808.

11. Pezet S, Malcangio M, Mcmahon SB. BDNF: a neuromodulator in nociceptive pathways? Brain Res Brain Res Rev 2002; 40(1-3):240-9. doi: 10.1016/s01650173(02)00206-0.

12. Fukuoka T, Kondo E, Dai $Y$, et al. Brain-derived neurotrophic factor increases in the uninjured dorsal root ganglion neurons in selective spinal nerve ligation model. J Neurosci 2001; 21(13):4891-900. doi: 10.1523/JNEUROSCI.21-13-04891.2001.

13. Wang $X$, Ratnam J, Zou B, et al. TrkB signaling is required for both the induction and maintenance 
of tissue and nerve injury-induced persistent pain. J Neurosci 2009; 29(17):5508-15. doi: 10.1523/ jneurosci.4288-08.2009.

14. Zhou XF, Deng YS, Xian CJ, et al. Neurotrophins from dorsal root ganglia trigger allodynia after spinal nerve injury in rats. Eur J Neurosci 2000; 12(1):100-5. doi: 10.1046/j.1460-9568.2000.00884.x.

15. de Oliveira Martins D, Martinez Dos Santos F, Evany De Oliveira $M$, et al. Laser therapy and pain-related behavior after injury of the inferior alveolar nerve: possible involvement of neurotrophins. J Neurotrauma 2013; 30(6):480-6. doi: 10.1089/neu.2012.2603.

16. Buchaim RL, Andreo JC, Barraviera B, et al. Effect of low-level laser therapy (LLLT) on peripheral nerve regeneration using fibrin glue derived from snake venom. Injury 2015; 46(4):655-60. doi: 10.1016/j.injury. 2015.01.031.

17. Dias FJ, Fazan VPS, Cury DP, et al. Growth factors expression and ultrastructural morphology after application of low-level laser and natural latex protein on a sciatic nerve crush-type injury. 2019; 14(1):e210211. doi: $10.1371 /$ journal.pone.0210211.

18. Buchaim DV, Andreo JC, Ferreira Junior RS, et al. Efficacy of laser photobiomodulation on morphological and functional repair of the facial nerve. Photomed Laser Surg 2017; 35(8):442-9. doi: 10.1089/ pho.2016.4204.

19. Mashhoudi Barez M, Tajziehchi M, Heidari MH, et al. Stimulation effect of low level laser therapy on sciatic nerve regeneration in rat. J Lasers Med Sci 2017; 8(Suppl 1):S32-S7. doi: 10.15171/jlms.2017.s7.

20. Bloom JD, Bleier BS, Goldstein SA, et al. Laser facial nerve welding in a rabbit model. Arch Facial Plast Surg 2012; 14(1):52-8. doi: 10.1001/archfacial.2011.61.

21. Bhatt NK, Khan TR, Mejias C, et al. Nerve transection repair using laser-activated chitosan in a rat model. Laryngoscope 2017; 127(8):E253-E7. doi: 10.1002/ lary. 26583.

22. Curtis NJ, Owen E, Walker DM, et al. Comparison of microsuture, interpositional nerve graft, and laser solder weld repair of the rat inferior alveolar nerve. J Oral Maxillofac Surg 2011; 69(6):e246-e55. doi: 10.1016/j.joms.2011.02.007.

23. Menovsky T, Beek JF. Carbon dioxide laser-assisted nerve repair: effect of solder and suture material on nerve regeneration in rat sciatic nerve. Microsurgery 2003; 23(2):109-16. doi: 10.1002/micr. 10100.

24. Steel B. Lingual split versus surgical bur technique in the extraction of impacted mandibular third molars: a systematic review. Oral Surg Oral Med Oral Pathol Oral Radiol 2012; 114(3):294-302. doi: 10.1016/ j.tripleo.2011.07.028.

25. Kim YK, Kim SG, Kim JH. Altered sensation after orthognathic surgery. J Oral Maxillofac Surg 2011; 69(3):893-8. doi: 10.1016/j.joms.2010.10.025.

26. Mohajerani SH, Tabeie F, Bemanali M, et al. Effect of low-level laser and light-emitting diode on inferior alveolar nerve recovery after sagittal split osteotomy of the mandible. J Craniofac Surg 2017; 28(4):e408-e11. doi: $10.1097 /$ SCS. 0000000000002929 .

27. Gasperini G, de Siqueira ICR, Costa LR. Lower-level laser therapy improves neurosensory disorders resulting from bilateral mandibular sagittal split osteotomy: a randomized crossover clinical trial. J Craniomaxillofac Surg 2014; 42(5):e130-e3. doi: 10.1016/ j.jcms.2013.07.009.

28. Fuhrer-Valdivia A, Noguera-Pantoja A, Ramirez-Lobos $V$, et al. Low-level laser effect in patients with neurosensory impairment of mandibular nerve after sagittal split ramus osteotomy. Randomized clinical trial, controlled by placebo. Med Oral Patol Oral Cir Bucal 2014; 19(4):e327-e34. doi: 10.4317/medoral.19626.

29. Guarini D, Gracia B, Ramírez-Lobos V, et al. Laser biophotomodulation in patients with neurosensory disturbance of the inferior alveolar nerve after sagittal split ramus osteotomy: a 2-year follow-up study. Photomed Laser Surg 2018; 36(1):3-9. doi: 10.1089/ pho.2017.4312.

30. Schultze-Mosgau S, Reich RH. Assessment of inferior alveolar and lingual nerve disturbances after dentoalveolar surgery, and of recovery of sensitivity. Int J Oral Maxillofac Surg 1993; 22(4):214-7. doi: 10.1016/s0901-5027(05)80638-1.

31. Pol R, Gallesio G, Riso M, et al. Effects of superpulsed, low-level laser therapy on neurosensory recovery of the inferior alveolar nerve. J Craniofac Surg 2016; 27(5):1215-9. doi: $10.1097 /$ SCS.0000000000002757.

32. Spencer $C R$, Irving $R M$. Causes and management of facial nerve palsy. $\mathrm{Br}$ J Hosp Med (Lond) 2016; 77(12):686-91. doi: 10.12968/hmed.2016.77.12.686.

33. Ferreira M, Marques EE, Duarte JA, et al. Physical therapy with drug treatment in Bell palsy: a focused review. Am J Phys Med Rehabil 2015; 94(4):331-40. doi: $10.1097 / \mathrm{phm} .0000000000000255$.

34. Holland NJ, Bernstein JM. Bell's palsy. BMJ Clin Evid 2014; 2014:1204.

35. Lampert L, Wong YJ. Combined antiviral-corticosteroid therapy for Bell palsy yields inconclusive benefit. J Am 
Dent Assoc 2012; 143(1):57-8. doi: 10.14219/jada. archive.2012.0020.

36. Fontana CR, Bagnato VS. Low-level laser therapy in pediatric Bell's palsy: case report in a three-year-old child. J Altern Complement Med 2013; 19(4):376-82. doi: 10.1089/acm.2011.0531.

37. Ordahan B, Karahan AY. Role of low-level laser therapy added to facial expression exercises in patients with idiopathic facial (Bell's) palsy. Lasers Med Sci 2017; 32(4):931-6. doi: 10.1007/s10103-017-21959.

38. Alayat MSM, Elsodany AM, El Fiky AAR. Efficacy of high and low level laser therapy in the treatment of Bell's palsy: a randomized double blind placebo-controlled trial. Lasers Med Sci 2014; 29(1):335-42. doi: 10.1007/s10103-013-1352-z. 